\title{
Investigating Patient Attitudes Towards the use of Social Media Data to Augment Depression Diagnosis and Treatment: a Qualitative Study
}

\author{
Jude Mikal \\ Minnesota Population Center \\ University of Minnesota \\ Minneapolis, MN, USA \\ jpmikal@umn . edu
}

\author{
Samantha Hurst \\ Family Medicine \& Public Health \\ University of California San Diego \\ La Jolla, CA, USA \\ shursteucsd.edu
}

\author{
Mike Conway \\ Biomedical Informatics \\ University of Utah \\ Salt Lake City, UT, USA \\ mike.conwaydutah.edu
}

\begin{abstract}
In this paper, we use qualitative research methods to investigate the attitudes of social media users towards the (opt-in) integration of social media data with routine mental health care and diagnosis. Our investigation was based on secondary analysis of a series of five focus groups with Twitter users, including three groups consisting of participants with a self-reported history of depression, and two groups consisting of participants without a selfreported history of depression. Our results indicate that, overall, research participants were enthusiastic about the possibility of using social media (in conjunction with automated Natural Language Processing algorithms) for mood tracking under the supervision of a mental health practitioner. However, for at least some participants, there was skepticism related to how well social media represents the mental health of users, and hence its usefulness in the clinical context.
\end{abstract}

\section{Introduction}

The widespread use of social media - including Twitter, Facebook, and online discussion forums such as Reddit - in combination with the maturation of technologies like Natural Language Processing (NLP) and Machine Learning has led to an increasing use of social media in population health research, with applications in infectious disease surveillance (e.g. Signorini et al. (2011); Collier et al. (2008); Freifeld et al. (2008)), understanding health behaviours and risk factors (e.g.
Hanson et al. (2013); Alvaro et al. (2015); Powell et al. (2016)), and investigating public attitudes towards health topics (e.g. Myslín et al. (2013); Oscar et al. (2017); Surian et al. (2016)). In addition to its proven utility for addressing research questions in population health, social media may also have considerable potential to enhance clinical care, particularly mental health care, by providing frequent, naturalistic, behavioural data that can be used by mental health practitioners to track moods and symptoms over time, allowing mental health clinicians to triangulate diagnoses and to better understand patient progress between appointments, hence improving quality of care.

In this paper, we use qualitative research methods to investigate the attitudes of social media users to the (opt-in) integration of social media data with routine mental health care and diagnosis. Our investigation was based on the secondary analysis of a series of five focus groups with Twitter users, conducted by author JM. Three of the groups were made up of participants with a diagnosed history of depression, and two of the groups were made up of participants without a diagnosed history of depression. These focus groups concentrated on ethical issues in utilising social media for population health monitoring (as reported in Mikal et al. (2016)), but also covered several related areas, including integrating automated analysis of social media data with routine mental health care. We presented Twitter users with the idea of allowing, with consent, mental health practitioners access to their patients' social media data in order to track mood over time, and ultimately improve care quality.

Our results indicate that, overall, research par- 
ticipants were enthusiastic about the possibility of using social media (in conjunction with automated NLP algorithms) for mood tracking in the context of a therapeutic relationship with a mental health practitioner. However, for at least some participants, there was skepticism related to how well social media represents the mental health of users.

\section{Background}

\subsection{Public Mental Health Research \& Social Media}

Social media has become an increasingly important resource for population level mental health research (Conway and O'Connor, 2016) with data sources including Reddit (e.g. Chen et al. (2015)), Twitter (e.g. Coppersmith et al. (2014)), and Facebook (e.g. Park et al. (2014)). Applications have included investigating new mothers' experiences of postpartum depression (De Choudhury et al., 2014), analysing language patterns associated with schizophrenia (Mitchell et al., 2015), examining the role of age and gender in tweeting about mental illness (Preoţiuc-Pietro et al., 2015), and tracking suicide risk factors (Jashinsky et al., 2014). Focussing specifically on major depressive disorder - one of the most common forms of mental illness with a lifetime prevalence of $16.2 \%$ (Kessler et al., 2003) - has been work on using computational methods for detecting changes in degree of depression based on Facebook status updates (Schwartz et al., 2014), and using unsupervised Machine Learning techniques to explore depression-related language on Twitter (Resnik et al., 2015).

\subsection{Combining Electronic Health Record Data with Social Media}

There is little research on public attitudes towards combining social media with Electronic Health Record (EHR) data for research and clinical care. A notable exception is Padrez et al. (2015), who in the context of a large, urban academic medical center in the United States - sought consent from 5256 "walk in" Emergency Room (ER) patients to link their social media (Facebook and Twitter) accounts with both their ER visit report, and their longitudinal EHR. Over one third of "walk-in" ER patients consented to this data linkage, indicating that at least for some social media users in some contexts, privacy concerns are not a barrier to linking EHR and social media data in the context of research. However, the research was not explicitly focussed on mental health, and users may feel particularly sensitive regarding the use of their mental health data for research purposes.

\section{Methods}

Qualitative data used in this study is derived from a series of five focus groups conducted between March and April 2015 by author JM (reported in Mikal et al. (2016)), with the principal purpose of exploring the ethical implications of using Twitter for population-level mental health monitoring. We opted for the use of focus groups to encourage the spontaneous generation of ideas through group interaction. Focus groups are considered to be an ideal method for the exploration of new ideas, and have the additional benefit that - unlike standard interviews - they emphasise interactions between participants and de-emphasise the role of the interviewer (Kitzinger, 1995). The first two focus group interviews were conducted with individuals with no diagnosed history of depression, while the subsequent three were conducted with individuals with a diagnosed history of depression In total, 26 participants were recruited (average age: 26.9; age range: $18-54 ; 2: 1$ male:female ratio - see Table 1 for participant characteristics). Focus groups were conducted face-to-face, and lasted two hours each - as is typical for focus group studies (Kitzinger, 1995). Interactions were audio-recorded and transcribed using a professional, HIPAA-compliant ${ }^{1}$ transcription service.

Each focus group began with participants introducing themselves. Control group participants stated their name (or pseudonym), age, occupation, and general Twitter use habits. Participants with depression also provided information on their depression history they were comfortable sharing: including diagnosis, medication, and therapy.

Qualitative coding was conducted manually (i.e. without the aid of qualitative analysis software like NVivo or ATLAS.ti) by author JM, then authors $\mathrm{JM}$ and MC met to discuss emergent themes. We used an inductive technique to allow themes to emerge from the data itself, guided by our research foci (Boeije, 2002).

\footnotetext{
${ }^{1}$ The Health Insurance Portability and Accountability Act (HIPAA) stipulates security standards for protected health information in the United States.
} 
Table 1: Participant characteristics

\begin{tabular}{|l|c|c|}
\hline Group & Age & Sex \\
\hline FG1:Control & 27 & $\mathrm{M}$ \\
FG1:Control & 22 & $\mathrm{M}$ \\
FG1:Control & 26 & $\mathrm{~F}$ \\
FG1:Control & 19 & $\mathrm{M}$ \\
FG1:Control & 22 & $\mathrm{~F}$ \\
\hline FG2:Control & 29 & $\mathrm{M}$ \\
FG2:Control & 21 & $\mathrm{~F}$ \\
FG2:Control & 21 & $\mathrm{~F}$ \\
FG2:Control & 40 & $\mathrm{~F}$ \\
FG2:Control & 24 & $\mathrm{M}$ \\
\hline FG3:Depression & 29 & $\mathrm{M}$ \\
FG3:Depression & 20 & $\mathrm{M}$ \\
FG3:Depression & 29 & $\mathrm{M}$ \\
FG3:Depression & 54 & $\mathrm{M}$ \\
\hline FG4:Depression & 42 & $\mathrm{M}$ \\
FG4:Depression & 21 & $\mathrm{~F}$ \\
FG4:Depression & 23 & $\mathrm{M}$ \\
FG4:Depression & 33 & $\mathrm{M}$ \\
\hline FG5:Depression & 20 & $\mathrm{~F}$ \\
FG5:Depression & 18 & $\mathrm{M}$ \\
FG5:Depression & 30 & $\mathrm{M}$ \\
FG5:Depression & 22 & $\mathrm{M}$ \\
FG5:Depression & 22 & $\mathrm{M}$ \\
FG5:Depression & 21 & $\mathrm{M}$ \\
FG5:Depression & 24 & $\mathrm{~F}$ \\
FG5:Depression & 31 & $\mathrm{M}$ \\
\hline
\end{tabular}

\section{Results}

\subsection{Therapeutic Utility of Social Media Data}

The possibility of using social media data under the supervision of a qualified mental health practitioner met with marked enthusiasm and approval in our focus groups. Participants reported that their mood and state of mind fluctuated over time, and that social media data could provide a more accurate assessment of their emotional state. When presented with the idea, Laurence ${ }^{2}$, a participant from one of our depression groups, had the following exchange with James, another participant:

Laurence: I think that sounds great! Especially I think one of the common questions is like, "How long have you felt this way?" "I don't know. I don't know."

James: Right, exactly. Forever.

\footnotetext{
${ }^{2}$ Note that all participant names are pseudonyms
}

Laurence: But if you could look at Twitter and just immediately [generate] a graph that shows mood swings over time. Absolutely!

Michael - a participant in a different depression group - similarly questioned his ability to accurately summarise his general state of mind between appointments, particularly if a significant amount of time had passed since his previous visit. When presented with the idea of having his mental health practitioner access his social media data, Michael says:

I'm all for that, because I know like when I've gone to therapists or my doctor or whatever, like I'm not the best at reporting how I've been doing. Like when I'm actually in an appointment. Especially like to go see them for the first time. Or to see them after I haven't seen them for a while. Like that would be fantastic to have something else to either support what I think, or to actually say, "Hey, you actually are going through something right now, and you should probably get some help for that." Just because I'm not reliable about accurately assessing how I'm doing.

Overall, research participants appreciated the potential use of social media data to confirm or contradict self-assessments, or to provide concrete evidence of emotional ups and downs in their dayto-day lives that they may not be able to recall when speaking with a therapist. In addition to objective mood assessment, social media data may help practitioners to pick up on cues that may be lost or ignored in peer-flagging programs:

Joe: Oh, I was just going to say- this probably makes me a bad person- but whenever I get the vague like "My life is terrible" Facebook posts, I just unfollow that person.

Lori: Seriously. They just want the attention.

Sara: I just wish there was an eye-roll button.

As summarized above, members of a peer network may not reach out in the instance of mental distress - or may block or choose to unfollow 
certain members of their peer groups because of the emotional ups and downs that may signify distress. Another advantage of algorithm-based social media analysis use in conjunction with a mental health practitioner is that algorithms and mental health providers may pay attention where the attention of peers may falter.

\subsection{Social Media and Self Presentation}

Results indicated that most participants felt as though their moods would be evident from their social media postings. For example when asked if his mental state would be evident from the data he generated on social media, Karl reported:

Yeah. For sure ...like my senior year, like I would just tweet just because I wanted my friends to see it, and to know that I didn't feel good, or that I was upset or mad at someone. And I definitely remember like going to Twitter to complain about people, or complain about how I felt. Or complain about like my day, or just say that I feel like shit, you know? I think it would be very obvious, actually.

When asked if the tweets would create an $\mathrm{accu}$ rate assessment of his mental state, Karl states:

I think they would probably be a little exaggerated, honestly, if I was to like look through them now, I would probably be like embarrassed at some of the shit that I said on the internet. Just like not thinking that it could go where it could go almost. But at the time, when you're just like in that fog, and like can't make yourself get out of bed, or don't want to do anything. Like just kind of having somewhere to like just send your thoughts was nice.

Interestingly, while Karl indicates that the tweets might present an exaggerated depiction of his depression, the tweets came at a time when he felt he that he was in a fog and was unable to get out of bed: likely signs of depression. This conflicting account of his "exaggerated" tweets during a time he would likely identify as having been depressed - illustrates the point made above: that social media may provide a more objective account, or at least another account of his feelings during this time that may be used to assess whether he was depressed or not.

Other focus group participants reported that not only were their own moods evident from social media posting, but that they could observe the moods of their own friends. For example, one of our focus group participants, Steve, worked in student affairs at a local university and said that during certain times of year it was possible to see an overall decline in student mood,

If you look at a student's Facebook or Twitter, especially like during finals time, you see how stressed people are. You see people aren't sleeping. They aren't eating and all they're doing is studying. And their moods are just getting worse and worse on social media.

Another participant, Dave, from the same focus group, who worked as a student life peer advisor echoed this view. Dave says that looking at the activities friends post about on Twitter may help give insight into their current state of being:

I mean you can tell when people are in certain moods on Twitter. Like if somebody was tweeting that they're watching a lot of Netflix and sitting around a lot - where they used to be outside or walking their dog, you can see a physical progression of the change through their Twitter. The cloud just gets darker as we progress in Twitter.

Additional information on sleep patterns, eating habits, and physical activity may provide helpful insights to mental health care providers to determine not only whether individuals are suffering from depression, but also whether the depression may be exacerbated by health-related behaviours.

Nevertheless, in discussing the accuracy of information shared on social media, the participants were mostly divided. For some, what they shared with a therapist in the context of a therapy session was more likely to be guarded or censored if they are not yet comfortable with the therapist. According to John:

[On social media], it's like you're in your natural environment. If I'm ever going to talk to [a therapist] I'm going to talk to them differently than I normally am because I'm not going to feel 
comfortable with them. But that's not going to cross my mind if I want to tweet something or if I want to Facebook something.

Conversely, some participants stated that they were unlikely to post about the things that were making them the most depressed. For them, social media data focuses on current events in their own lives and across the world. When asked if she thought her mood would be evident from her social media behaviour, Katy responds:

No. Because I don't post super often and even when I do it usually does not really reflect my mood. It's usually like news or reaction to some kind of other thing that I've seen online or like a picture of my cat. Any kind of psychologist would not be able to see what I'm actually feeling because that's not something that I feel the need to express online. How I'm feeling goes into my physical diary.

According to another participant, not all social media accounts are created equal and what a therapist might surmise using her Twitter account data is not the same as what a therapist might surmise from her Facebook data. Cassie summarizes as follows,

[When posting on Facebook], it doesn't matter if I'm happy or sad. You can't see - there she got a scholarship or oh she didn't get a scholarship. You can't see that a credit card bill was late. You don't see any of the things that are bummers. It's all just like look at what I ate, this is where I was. But Twitter might be better - because my Twitter is more like when I do post on Twitter it's a little bit more expressive because it's just thoughts. So it's like oh I'm really bummed right now.

In light of this, when evaluating social media data for evidence of depression or mental health dysfunction, it may be important to ask individuals who are seeking help both whether their accounts would provide any useful insight into their state of mind, and which account would provide the most accurate assessment. In addition, it may be helpful to have individuals flag certain events that may have triggered a depression episode.

Other concerns highlighted by participants centred on how often users posted and how accurately they portrayed themselves. Individuals' Twitter use varied from those who were occasional tweeters, to individuals who maintained upwards of ten Twitter profiles and tweeted multiple times per day. According to one participant, Bob, the data generated by more active users was more likely to provide an accurate assessment of mental distress than social media data generated by more passive users. Bob says:

Your accuracy level is very much going to depend on the activity of the user. For example, [...] if your psychiatrist or your therapist had access to your entire process, and they could see that you have an increased amount of depression Tweets during winter, immediately they can say, okay, "Well, possible Seasonal Depressional Disorder." You know, based on that access. They look at mine, who the hell- I'm so all over Twitter, nobody's going to have any idea, because I'm not a regular user. So [I] think that's definitely going to have to play in.

Additionally, users reported that they were careful to manage their self-presentation on Twitter. Sara, a stay at home parent, reported that she only says positive things on Twitter. According to another participant, Sara's experiences may not be the exception. For Karen, social media is about explicitly presenting a persona (Goffman, 1971) and as such, would be of little help in diagnosing mental distress. Karen says:

Yeah, but like at the same time, I feel like people are really big on making themselves sound more interesting on social networks. Ever since social networks became huge I feel like people have this image. What's said between like me and my therapist, that's like the full, raw details, but on the Internet I could just be like, "Oh, I went to this one show," and people will think I'm fine. But what I'm telling my therapist is, like, the exact opposite. 


\section{Limitations}

This study has several limitations. First, the results are qualitative in nature, and based on the secondary analysis of focus group data, hence generalisability is limited. Second, our participants were all drawn from a relatively socially conservative region of the western United States. Third, our recruitment method relied on advertising on a local community Reddit site, and therefore our sample - like Reddit - skewed young and male (Pew Research Center, 2016).

\section{Conclusions}

In conclusion, we have explored two broad areas that have emerged from focus group discussions with respect to using automated analysis of social media data to enhance mental health care: therapeutic utility of social media data and social media $\&$ self presentation. Note that while there were some doubts expressed concerning the ability of NLP algorithms to successfully identify mental status from social media data (i.e. a technological limitation) most of the discussion around accuracy centred on questions of self-presentation in social media. Overall, participants were enthusiastic about the idea of opt-in utilisation of social media in the context of clinician-led mental health care, but at least for some participants, there was some skepticism related to how well social media represents the mental health of users.

\section{Acknowledgments}

We would like to thank Dr Daniel O'Connor (Wellcome Trust) for valuable feedback in the early stages of this research.

Research reported in this publication was supported the National Library of Medicine (United States National Institutes of Health) under award numbers K99LM011393 and R00LM011393. The content is solely the responsibility of the authors and does not necessarily represent the official views of the National Institutes of Health. Note that the real names of participants have been replaced by pseudonyms in order to protect participant anonymity.

\section{Ethical Approval}

The research reported in this paper was approved by the University of Utah Institutional Review Board [Ethics Committee] (\#00077913).

\section{References}

Nestor Alvaro, Mike Conway, Son Doan, Christoph Lofi, John Overington, and Nigel Collier. 2015. Crowdsourcing Twitter annotations to identify first-hand experiences of prescription drug use. J Biomed Inform 58:280-7. https://doi.org/10.1016/j.jbi.2015.11.004.

Hennie Boeije. 2002. A purposeful approch to the constant comparitive method in the analysis of qualitative interviews. Qual Quant 36:391-409.

Annie T Chen, Shu-Hong Zhu, and Mike Conway. 2015. What online communities can tell us about electronic cigarettes and hookah use: A study using text mining and visualization techniques. J Med Internet Res 17(9):e220. https://doi.org/10.2196/jmir.4517.

Nigel Collier, Son Doan, Ai Kawazoe, Reiko Matsuda-Goodwin, Mike Conway, Yoshio Tateno, Quoc-Hung Ngo, Dinh Dien, Asanee Kawtrakul, Koichi Takeuchi, Mika Shigematsu, and Kiyosu Taniguichi. 2008. BioCaster: Detecting Public Health Rumors with a Web-based Text Mining System. Bioinformatics 24(24):2940-2941. https://doi.org/10.1093/bioinformatics/btn534.

Mike Conway and Daniel O'Connor. 2016. Social media, big data, and mental health: Current advances and ethical implications. Current Opinion in Psychology 9:77-82. https://doi.org/10.1016/j.copsyc.2016.01.004.

Glen Coppersmith, Mark Dredze, and Craig Harman. 2014. Quantifying mental health signals in Twitter. In Proceedings of the Workshop on Computational Linguistics and Clinical Psychology: From Linguistic Signal to Clinical Reality. Association for Computational Linguistics, Baltimore, Maryland, USA, pages 51-60. http://www.aclweb.org/anthology/W/W14/W143207.

Munmun De Choudhury, Scott Counts, Eric Horvitz, and Aaron Hoff. 2014. Characterizing and predicting postpartum depression from shared facebook data. In Computer Supported Cooperative Work, CSCW '14, Baltimore, MD, USA, February 15-19, 2014. ACM, pages 626-638. http://doi.acm.org/10.1145/2531602.2531675.

Clark C Freifeld, Kenneth D Mandl, Ben Y Reis, and John S Brownstein. 2008. Healthmap: global infectious disease monitoring through automated classification and visualization of internet media reports. J Am Med Inform Assoc 15(2):150-7. https://doi.org/10.1197/jamia.M2544.

Erving Goffman. 1971. The presentation of self in everyday life. A Pelican book. Penguin, Harmondsworth.

Carl L Hanson, Scott H Burton, Christophe GiraudCarrier, Josh H West, Michael D Barnes, and Bret 
Hansen. 2013. Tweaking and tweeting: exploring Twitter for nonmedical use of a psychostimulant drug (adderall) among college students. J Med Internet Res 15(4):e62.

Jared Jashinsky, Scott H Burton, Carl L Hanson, Josh West, Christophe Giraud-Carrier, Michael D Barnes, and Trenton Argyle. 2014. Tracking suicide risk factors through Twitter in the US. Crisis 35(1):51-9.

Ronald C Kessler, Patricia Berglund, Olga Demler, Robert Jin, Doreen Koretz, Kathleen R Merikangas, A John Rush, Ellen E Walters, Philip S Wang, and National Comorbidity Survey Replication. 2003. The epidemiology of major depressive disorder: results from the national comorbidity survey replication (NCS-R). JAMA 289(23):3095-105. https://doi.org/10.1001/jama.289.23.3095.

Jenny Kitzinger. 1995. Qualitative research. introducing focus groups. BMJ 311(7000):299-302.

Jude Mikal, Samantha Hurst, and Mike Conway. 2016. Ethical issues in using Twitter for population-level depression monitoring: a qualitative study. $B M C$ Med Ethics 17:22. https://doi.org/10.1186/s12910016-0105-5.

Margaret Mitchell, Kristy Hollingshead, and Glen Coppersmith. 2015. Quantifying the language of schizophrenia in social media. In Proceedings of the 2nd Workshop on Computational Linguistics and Clinical Psychology: From Linguistic Signal to Clinical Reality. Association for Computational Linguistics, Denver, Colorado, pages 11-20. http://www.aclweb.org/anthology/W15-1202.

Mark Myslín, Shu-Hong Zhu, Wendy Chapman, and Mike Conway. 2013. Using Twitter to examine smoking behavior and perceptions of emerging tobacco products. J Med Internet Res 15(8):e174.

Nels Oscar, Pamela A Fox, Racheal Croucher, Riana Wernick, Jessica Keune, and Karen Hooker. 2017. Machine learning, sentiment analysis, and tweets: An examination of Alzheimer's disease stigma on Twitter. J Gerontol B Psychol Sci Soc Sci https://doi.org/10.1093/geronb/gbx014.

Kevin A Padrez, Lyle Ungar, Hansen Andrew Schwartz, Robert J Smith, Shawndra Hill, Tadas Antanavicius, Dana M Brown, Patrick Crutchley, David A Asch, and Raina M Merchant. 2015. Linking social media and medical record data: a study of adults presenting to an academic, urban emergency department. BMJ Quality \& Safety https://doi.org/10.1136/bmjqs-2015-004489.

Gregory Park, H. Andrew Schwartz, Johannes C Eichstaedt, Margaret L Kern, Michal Kosinski, David J Stillwell, Lyle H Ungar, and Martin E P Seligman. 2014. Automatic personality assessment through social media language. Journal of Personality and Social Psychology 108(6):934-952.
Pew Research Center. 2016. Nearly eightin-ten Reddit users get news on the site. http://www.webcitation.org/6pQiU7EKp.

Gregory E Powell, Harry A Seifert, Tjark Reblin, Phil J Burstein, James Blowers, J Alan Menius, Jeffery L Painter, Michele Thomas, Carrie E Pierce, Harold W Rodriguez, John S Brownstein, Clark C Freifeld, Heidi G Bell, and Nabarun Dasgupta. 2016. Social media listening for routine postmarketing safety surveillance. Drug Saf 39(5):44354. https://doi.org/10.1007/s40264-015-0385-6.

Daniel Preoţiuc-Pietro, Johannes Eichstaedt, Gregory Park, Maarten Sap, Laura Smith, Victoria Tobolsky, H. Andrew Schwartz, and Lyle Ungar. 2015. The role of personality, age, and gender in tweeting about mental illness. In Proceedings of the 2nd Workshop on Computational Linguistics and Clinical Psychology: From Linguistic Signal to Clinical Reality. Association for Computational Linguistics, Denver, Colorado, pages 21-30. http://www.aclweb.org/anthology/W15-1203.

Philip Resnik, William Armstrong, Leonardo Claudino, Thang Nguyen, Viet-An Nguyen, and Jordan Boyd-Graber. 2015. Beyond LDA: Exploring supervised topic modeling for depression-related language in Twitter. In Proceedings of the $2 \mathrm{nd}$ Workshop on Computational Linguistics and Clinical Psychology: From Linguistic Signal to Clinical Reality. Association for Computational Linguistics, Denver, Colorado, pages 99-107. http://www.aclweb.org/anthology/W15-1212.

H. Andrew Schwartz, Johannes Eichstaedt, Margaret L. Kern, Gregory Park, Maarten Sap, David Stillwell, Michal Kosinski, and Lyle Ungar. 2014. Towards assessing changes in degree of depression through Facebook. In Proceedings of the Workshop on Computational Linguistics and Clinical Psychology: From Linguistic Signal to Clinical Reality. Association for Computational Linguistics, Baltimore, Maryland, USA, pages 118-125. http://www.aclweb.org/anthology/W/W14/W143214.

Alessio Signorini, Alberto Maria Segre, and Philip M Polgreen. 2011. The use of Twitter to track levels of disease activity and public concern in the U.S. during the influenza A H1N1 pandemic. PLoS One 6(5):e19467. https://doi.org/10.1371/journal.pone.0019467.

Didi Surian, Dat Quoc Nguyen, Georgina Kennedy, Mark Johnson, Enrico Coiera, and Adam G Dunn. 2016. Characterizing Twitter discussions about HPV vaccines using topic modeling and community detection. J Med Internet Res 18(8):e232. https://doi.org/10.2196/jmir.6045. 